\title{
Indoor motion analysis of a subject wearing prosthesis for adaptive snowboarding
}

\author{
L. Gastaldi ${ }^{1}$, S. Pastorelli ${ }^{1}$, M. Caramella ${ }^{2}$ \& U. Dimanico ${ }^{2}$ \\ ${ }^{I}$ Department of Mechanics, Politecnico di Torino, Italy \\ ${ }^{2}$ S.S.D. Neurofisiologia Riabilitativa ASL CN1, Fossano, Italy
}

\begin{abstract}
The growing popularity of adaptive snowboarding has motivated the designs of new prostheses for lower limb amputees. In the paper the biomechanics of an amputee subject wearing an energy-storing trans-femoral prosthesis for snowboarding is investigated. Experimental motion analysis lab tests were conducted by two expert surfers, an amputee and an able body subject, in order to compare the different behaviours.

Results for the two subjects and for different working conditions are reported and analysed. A strong dependence of the prosthesis behaviour on working conditions, especially on working frequency, can be pointed out.

Keywords: adaptive snowboard, movement analysis, lower limb prosthesis.
\end{abstract}

\section{Introduction}

It is well known that sport activity is an excellent functional and psychological rehabilitation for subject with limb capacity compromised due to traumatic events, such as amputees or spinal cord injured. Besides sport can also be a mean to overcome their present physical limitations and more in general physical activity presents many benefits, including a decrease in pain, depression and an increase in the quality of life.

Still a redefinition of the sports is necessary to make them adaptive, defining rules and athletes' classification, and finally re-designing equipments. While rules and classification are under the jurisdiction of international organisation and they must be as general as possible to consider all the disabilities, equipment matter is a more delicate aspect, as it has to respect general rules and at the same 
time it has to be personalised in order to fulfil functional and physical gaps that exist between athletes.

For amputees entry equipment encompasses also dedicated prostheses, as sport activity is more demanding, from a mechanical aspect, than every day activities. As a matter of facts even if we consider very sophisticated solutions, both from material or functions point of view, the prosthesis will never accomplish all the physiological tasks, such as providing shock absorption, knee stabilisation, limb length change in order to minimise the displacement of the centre of gravity, etc. Consequently, the necessity to understand the specific biomechanics of the sport and the physical characteristic of the remnant limb in order to design a specific solution and to enhance injured subjects to pursue athletic performances. The prosthesis is a tool that plays an important role in maximising performances, especially speaking of elite athletes.

Actually in the winter paralympic sports scenario adaptive snowboard is spreading more and more. National and International Federations are arising and the goal is the snowboard discipline debut in the next Winter Paralympic Games in 2014 and many snowboarders are dreaming about it. The introduction of this new sport discipline will also help to involve young people, which is a main task of all the disable committees.

Few studies can be found about biomechanics of the lower extremities in snowboarding, although no one is addressed to lower limb prostheses. Corresponding to the determination of kinematical parameters of the ankle joint complex, Delorme et al. [1] analysed this complex while on slope snowboarding. To improve safety aspects in skiing and snowboarding, Klous et al. [2] compared the loading at the knee and ankle joint in skiing and snowboarding. Interesting results about kinematics and kinetics are presented in [3], where ground reaction forces of jump landings in freestyle snowboarding are measured by means of a snowboard-mounted force platform. However, the mechanical characteristics (weight, standing height) of such a system can affect a snowboarder adversely. Finally, Krüger et al. in [4] present a pilot study to determine relevant kinematical and kinetic parameters using an inertial measurement suit in combination with a bilateral insole measurement system.

In skiing, several biomechanical studies roughly estimated joint loading in turning $[5,6]$ and on landing manoeuvres after a jump $[7,8]$, but none with sufficient accuracy.

Generally results show that loading is more evenly distributed between the legs in snowboarding than in skiing and that the highest moments were found in the rear leg in snowboarding.

As stated above the immense popularity of snowboarding has suggested the developmental designs of prostheses for trans-femoral amputees. The present study investigates performances of a trans-femoral prosthesis for snowboarding and in the paper biomechanical results from an indoor movement analysis are acquired and commented. These results are necessary for the further step that consists in an inverse dynamic study of the trans-femoral prosthesis and in evaluating forces in the joints and on the stump. 


\section{Adaptive snowboard}

Adaptive snowboard events include male and female athletes with a physical disability such as spinal injury, cerebral palsy, amputation, and visual impairments. Athletes compete based on their functional ability, allowing participants with different disabilities to race against each other.

Adaptive snowboarding athletes compete and use the same venue as ablebody snowboardcross events. The event format is also conducive to a classification process, which will be finalized by the adaptive snowboarding International Federation which is in working progress. Classification is a very delicate point, and it is undertaken to ensure that an athlete's impairment is relevant to sport performance and to ensure that the athlete competes equitably with all the other athletes. The process will held to define, for each category, a time factor that multiplies the real time achieved by the athletes. For non sever impairments the multiplier factor is equal to 1 and it decreases for severer impairments. The three macro-classes are: blind, standing and sitting, among which sub-categories are detected according to the disability level.

In general, snowboards are chosen on the basis of the rider's height, weight, and ability level. Some adaptive riders use outriggers to help balance themselves while they board, but many don't use any special equipment. Also bindings on the board can be moved to help with balance.
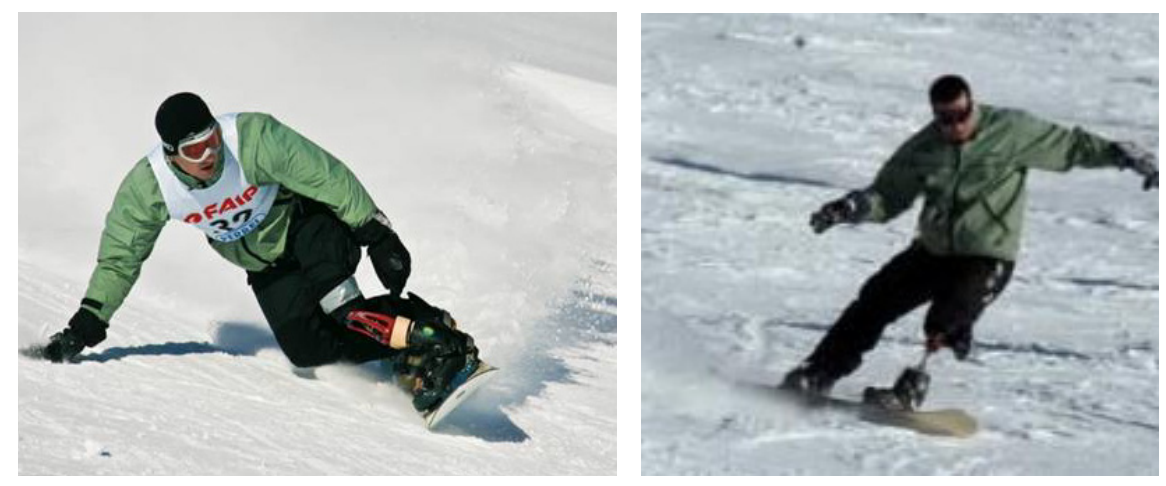

Figure 1: Adaptive snowboarding.

For the standing class, athlete must be able to keep her/his balance; this is possible moving conveniently the body center of mass and controlling the lower limb flexo-extension angles. The slow down phase is obtained by impressing a pressure with the front or rear part of the feet and concentrating the weight in the rear part of the snowboard. The breaking action is more effective as the snowboard axis and the course direction become more perpendicular. Pressure control is also used to control downhill velocity, while change of direction is managed rotating the upper part of the body. 
The rider, as the able body one, can assume two different positions, called Regular and Goofy. This refers to the dominant way surfer points the board down the hill. Regular means left foot forward, while goofy means right foot forward.

Rotation movements are basic and they can be distinct in backside or frontside. When in the first part of the spin the front part of the body faces the mountain, then this is a frontside or toe-side turn, the opposite is a back or heelside turn.

To surf it is necessary a prosthesis allowing a loaded, flexed knee and ankle position, as snowboarders are in bilateral dynamic hip, knee, and ankle flexion as they negotiate the hill.

\section{Experimental tests}

Due to the intrinsic difficulties in performing effective tests on the ski runs without influencing the natural surfing gesture, authors opted for a preliminary activity consisting in lab tests motion analysis. The indoor conditions do not allow us to reproduce exactly the surfing movement, thus a significant gesture has been taken into account to evaluate the biomechanics of the amputee athlete wearing the lower limb prosthesis with respect to an able-body subject.

Tests were carried out in a gait analysis lab in which kinematic and dynamic clinical analyses are usually performed.

\subsection{Prosthetic device}

The prosthesis used during tests is a commercial one specifically designed for snow sports. Knee and ankle joints are replaced by two planar cylindrical hinges that allow flexo-extension in the sagittal plane. To mimic the quadriceps and hamstring muscles a passive energy-storing system is present: a pneumatic spring and a hydraulic shock absorber are integrated into the prosthesis both at the knee and ankle level. The spring system stores energy when loaded during flexion and it returns it during extension to help the athlete to promptly regain an upright standing position. The damper provides an impact absorption capacity and it delivers less dangerous impact to the musculoskeletal system. The system stiffness is user-adaptable by changing spring air pressure and three damping sets are available.

To allow the user to wear a standard snowboard-boot and to use standard bindings, a customized foot is fixed to the prosthesis. During tests this had been replaced by a rigid insole as they were conducted by barefoot users.

\subsection{Experimental protocol}

Two subjects were selected, a left trans-femoral amputee and a healthy subject, in order to compare the different behaviours.

Subjects were request to repeat movements similar to the one performed on the ski slope; both are expert surfers and normally the amputee uses the 
prosthesis to ride. Tests consist of squat exercises executed with three one-byone changeable tests conditions:

- feet position. In figure 2 the reciprocal positions between feet axes and frontal plane are depicted. "Central position" corresponds to the physiological standing position in which feet are slightly externally rotated (about $7^{\circ}$ ) and they are symmetric respect to the sagittal plane. In "left position" subjects were requested to keep shoulders still, while feet were rotated about the vertical axis by $30^{\circ}$ anticlockwise. Similarly for the "right position", where rotation was $30^{\circ}$ clockwise.

- load. Subjects hold a barrel on the shoulders and performed tests without loads on the barrel itself or with an additional load equal to $20 \%$ of the subject's weight.

- squat frequency. Amputee subject was requested to squat at two selfchosen frequencies, a high (approx 140 beats/min) and a low (approx. 35 beats/min) one. The able-body subject fits his frequencies to the amputee's ones. No restriction was imposed on the pelvis vertical displacements.

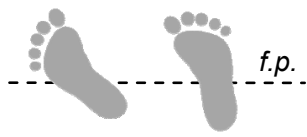

left position

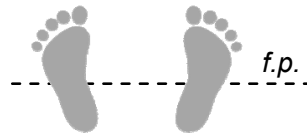

central position

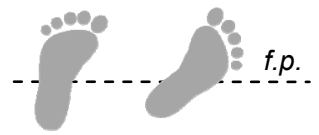

right position

Figure 2: Feet position with respect to the frontal plane (f.p.) during experimental tests.
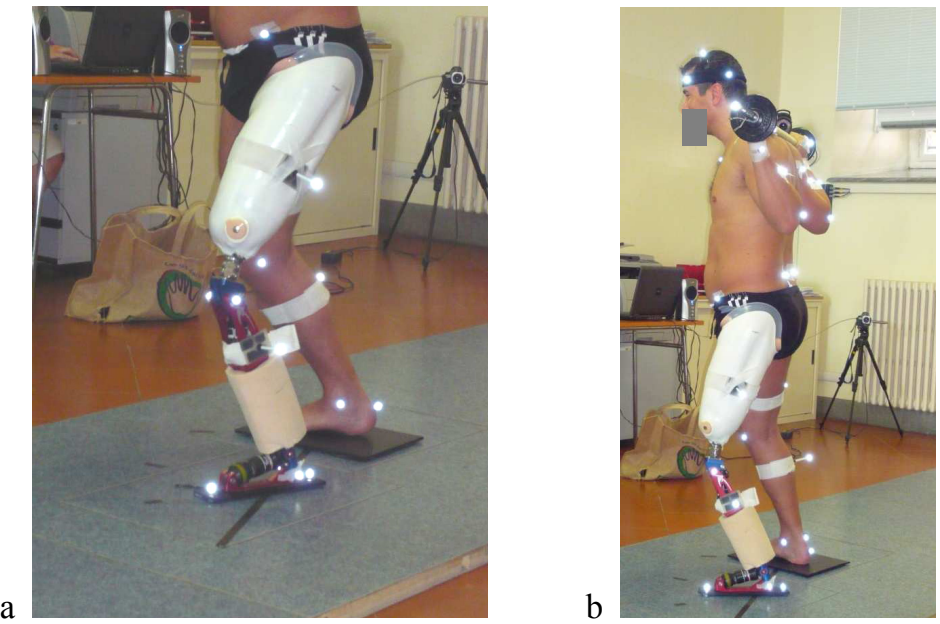

Figure 3: $\quad$ Experimental tests with the prosthesis. 


\subsection{Motion analysis system}

Movements were acquired using a stereo-photogrammetric system (VICON 6 infrared cameras with a 100 frames/second) and ground reaction forces were recorded using two six components force plates.

Markers were placed according the Plug-in-Gait protocol [9] for the healthy subject and according to a modified Plug-in-Gait protocol for the markers placed on the prosthesis, as it is pointed out in Figure 3a.

Markers were added also on the barrel and on the upper body in order to check the correctness of the squat movements (Figure 3b). Totally 51 markers were acquired and processed. After movements tests, from a full 3-D movement reconstruction kinematics of body and prosthesis is obtained.

\section{Results}

All the subjects' kinematics and ground reactions were analysed; however in the paper only main results are reported. For both limbs in the next figures following data trends are depicted:

- knee flexo-extension angles, ankle plantar and dorsi-flexion angles, which correspond to the degrees of freedom of the prosthesis;

- the vertical components of the ground reaction forces measured by the force platforms;

- the spatial position of the feet centre of pressure (COP) on the force plates; the diagonal line represents the subject frontal plane projection;

- picture of the subject during respective tests.

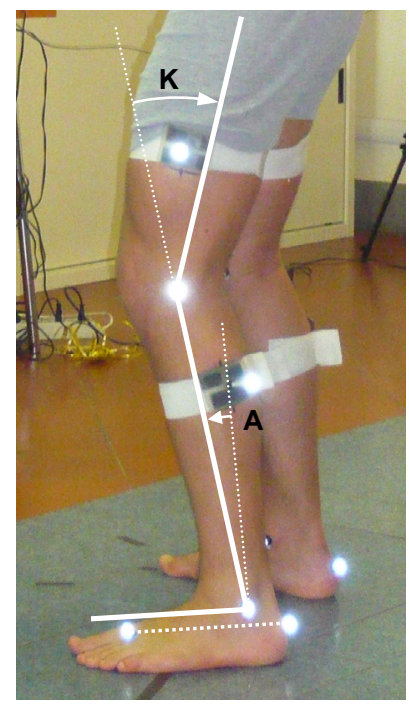

Figure 4: Convention to measure ankle $(\mathrm{A})$ and knee $(\mathrm{K})$ angles. 
The ankle plantar and dorsi-flexion is measured between the line perpendicular to the foot axis and the shank long axis; while the knee flexoextension angle is measured between the thigh and shank long axes. A $0^{\circ}$ of plantar flexion corresponds to shank and foot right angled, while a $0^{\circ}$ of knee flexion is measured when thigh and shank are aligned. In figure 4 the conventions adopted to measure knee (K) and ankle (A) angles are reported.

At first a comparison is made between the able-body and the amputee subject; then the prosthesis performances in different test conditions are analysed.

In Figures 5 and 6 results of the test performed at high frequency, with no load and with the feet in central position respectively for the able-body and the amputee subject are reported. These test configurations will be taken as standard, and these conditions will be changed one at a time in order to evaluate the influence of the single parameters on the complete movement performances.

In the case of Figure 5a good symmetry both for the kinematics and for the Ground Reaction Forces (GRFs) can be pointed out. Knees and ankles angles ranges are respectively about $25^{\circ}$ and $12^{\circ}$, while forces change between 280 and $700 \mathrm{~N}$. The Centre of Pressure (COP) location is concentrated in the central part of the feet.

Considering figure 6 , from the first graph it can be seen that the sound limb knee flexes for a greater angle $\left(15^{\circ}\right)$, the prosthesis knee angle has a more
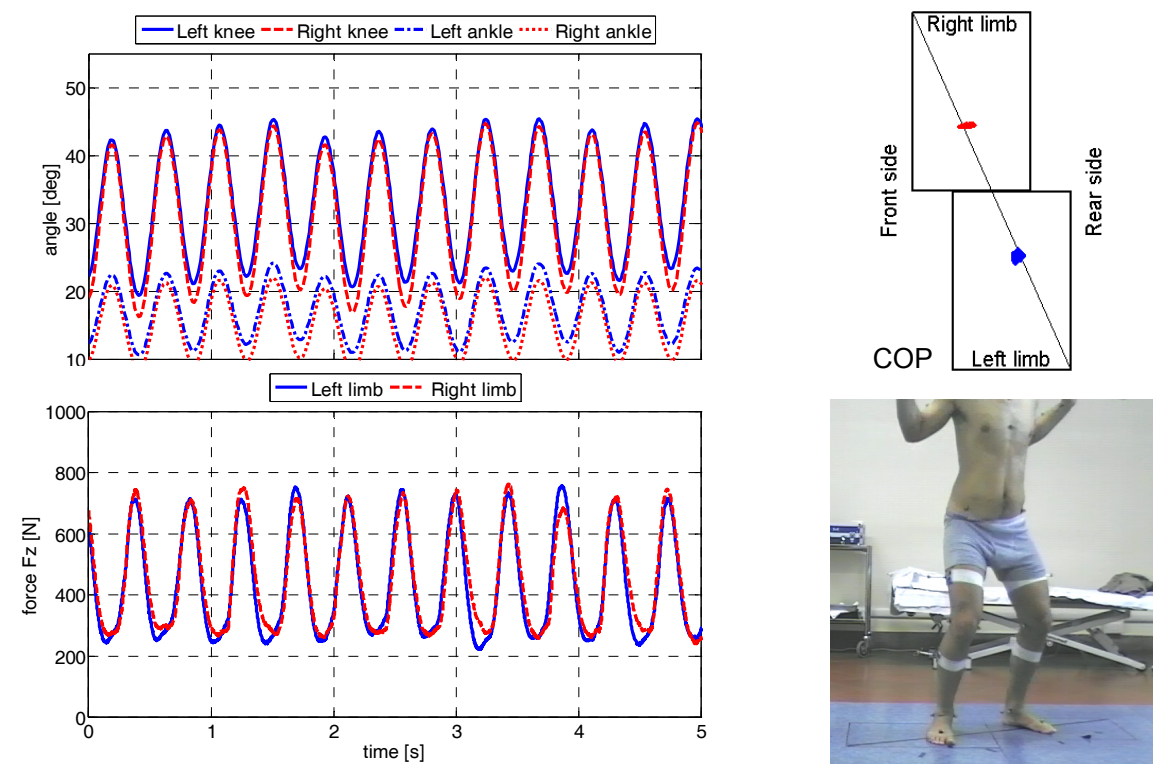

Figure 5: Able body subject. Test conditions: high frequency, no applied load and central feet position. 

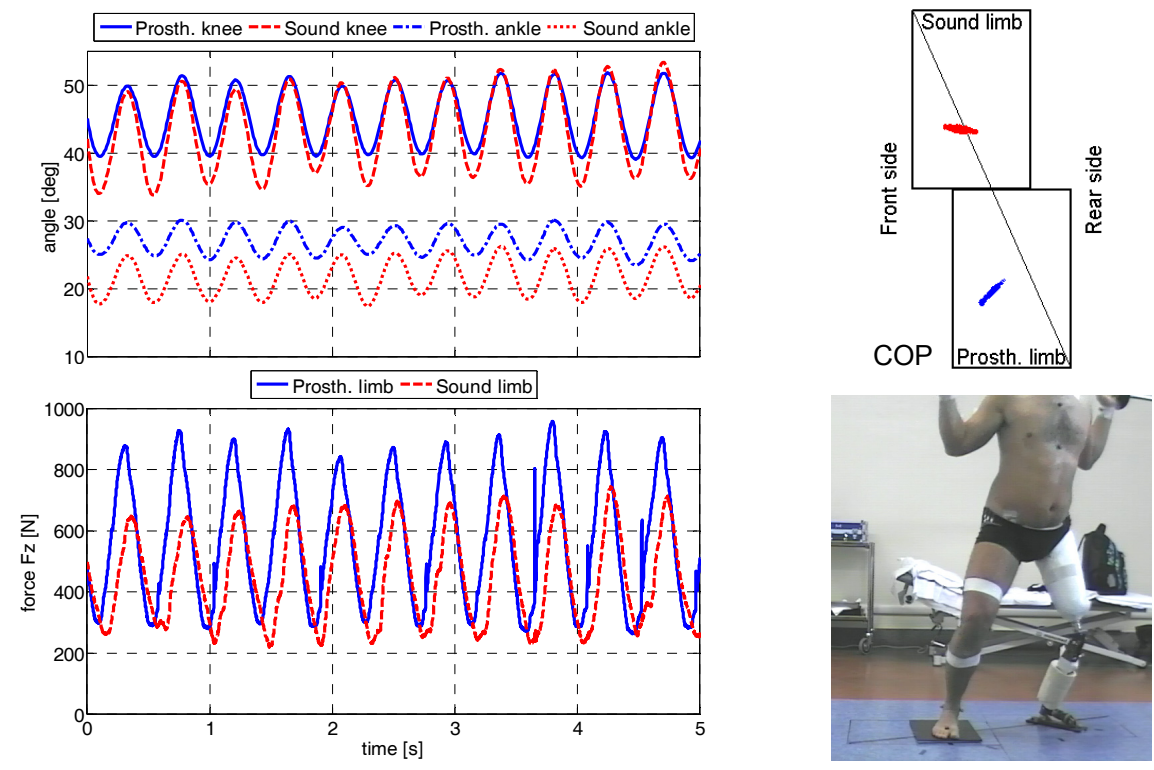

Figure 6: Amputee subject. Test conditions: high frequency, no applied load and central feet position.

limited range (approx. $10^{\circ}$ ), while the ankle flexion range is about $5^{\circ}$. For both joints it can be observed that the mean values of the flexion angles are higher for the prosthetic limb. This can be explained observing the GRFs, where the force exchanged with the ground is higher at the prosthesis side. In fact to obtain knee angles of $50^{\circ}$ subject needs to overload the prosthesis and hence to unload the sound limb.

Moreover, considering the displacement of the COP, there is a distribution of the point in which reaction forces are applied on all the contact area for the sound limb, while for the prosthetic one a concentration on the tip can be noticed.

For the able body subject the COP is concentrated in the middle of the foot, in correspondence of the frontal plane projection line. The area swept from the $\mathrm{COP}$ is in any case smaller for the able body subject than the amputee.

Trends reported in figure 7 were recorded during a test with the same conditions of the previous one, except for the frequency that this time was the low one.

In this case there is a major mobility of the prosthetic limb respect to the sound one, but these data need to be correlated with the ground reaction forces trends. Firstly it can be observed that the total ground reaction force (given by the sum of the two reactions) is diminished. In fact the slower movement involves minimal inertia actions; practically the instantaneous total force corresponds to the subject's weight. 
This requires a major translation of the body centre of mass on the prosthesis side. This is done by the subject and it can be seen from trends in Figure 6 where the two GRFs are in opposite phase. The effect is that the subject has his centre of mass totally unbalanced on the prosthesis and for these reasons the sound leg is in a more extended stance.

The COP is still concentrated on the tip of the sole and the area is more restricted than the previous case both for the sound and non sound limb.

The effect of foot position can be gather observing results reported in Figure 8. In this case foot are placed in a "left position" (Figure 2), corresponding to a regular rider, as the user effectively is.

In this case the body weight is naturally shifted on the front limb, which turns out also to be the more flexed one. This working configuration is particularly favourable for the prosthesis.

The knee joints excursion is almost the same for both legs, only the flexion angle is greater for the prosthetic limb. The minor flexion of the sound limb knee is compensated by the corresponding ankle that in this case is greater compared to the opposite one. For the GRFs it can be observed that a slight phase shift is present.

Interesting is the area swept by the COP. For the sound limb the area is reduced respect to the full footmark and the COP moves in the internal part of the foot, while concerning the prosthesis only the front part is loaded and the COP zone is smaller.
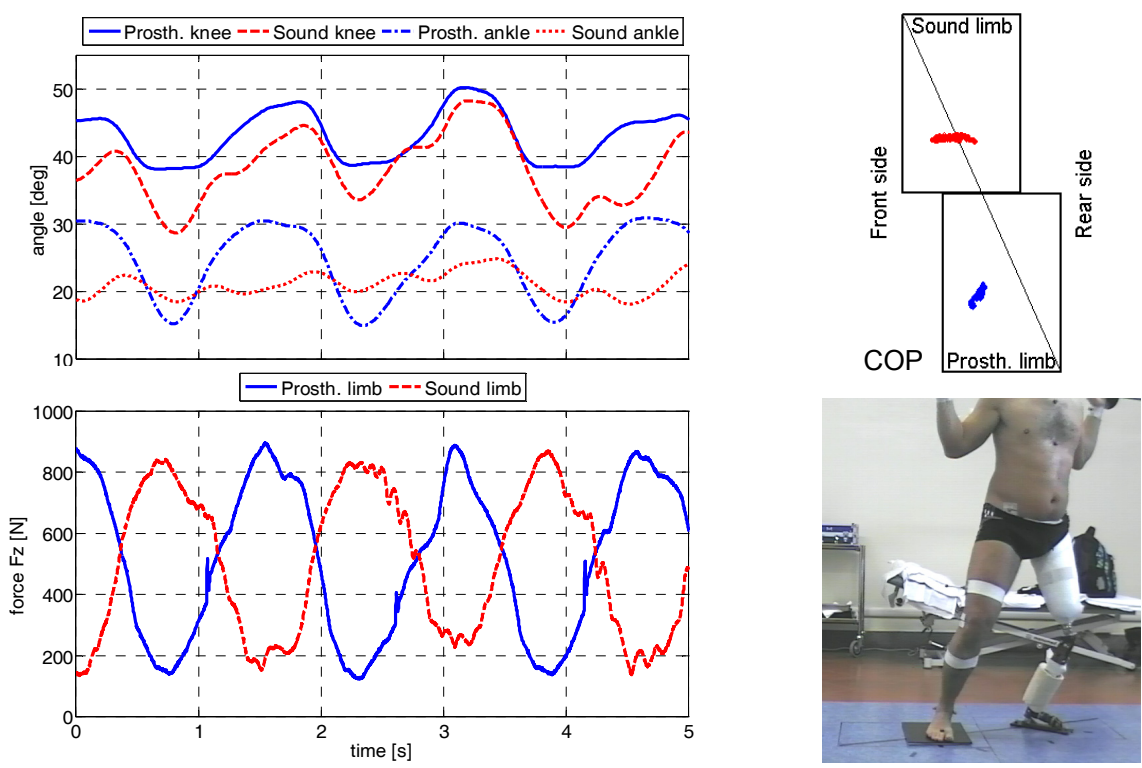

Figure 7: Amputee subject. Test conditions: low frequency, no applied load and central feet position. 

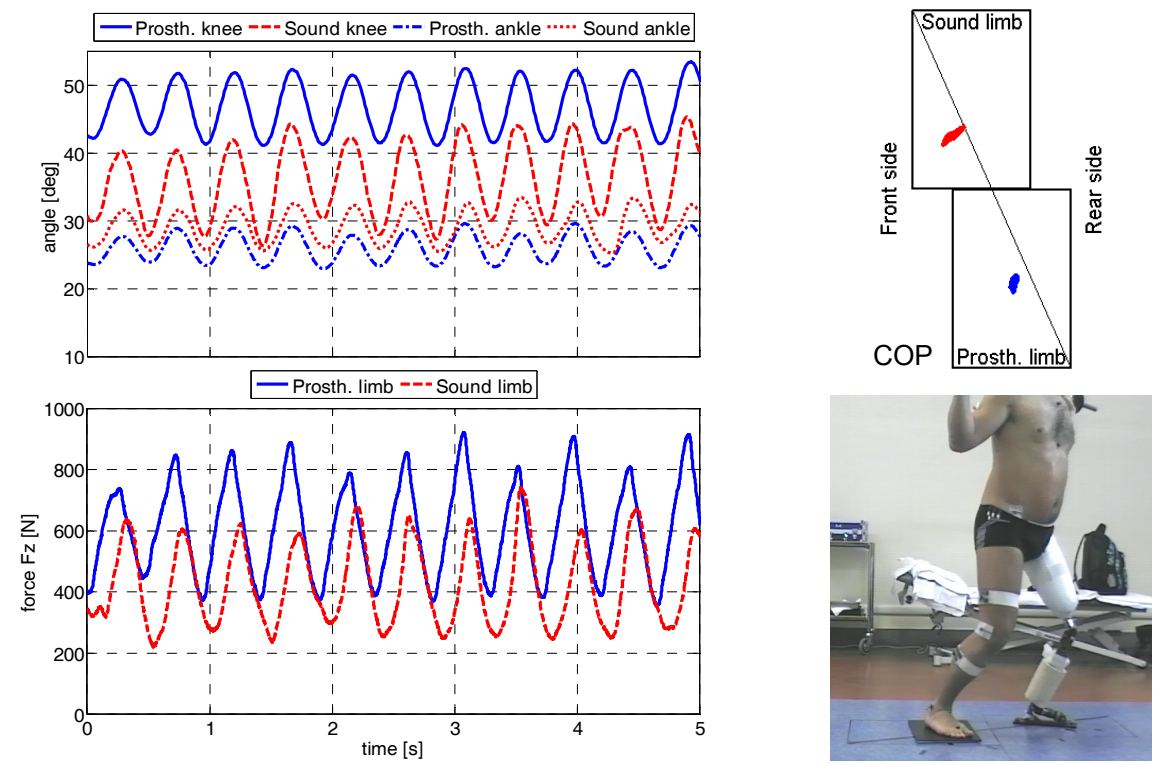

Figure 8: Amputee subject. Test conditions: high frequency, no applied load and feet in left position.
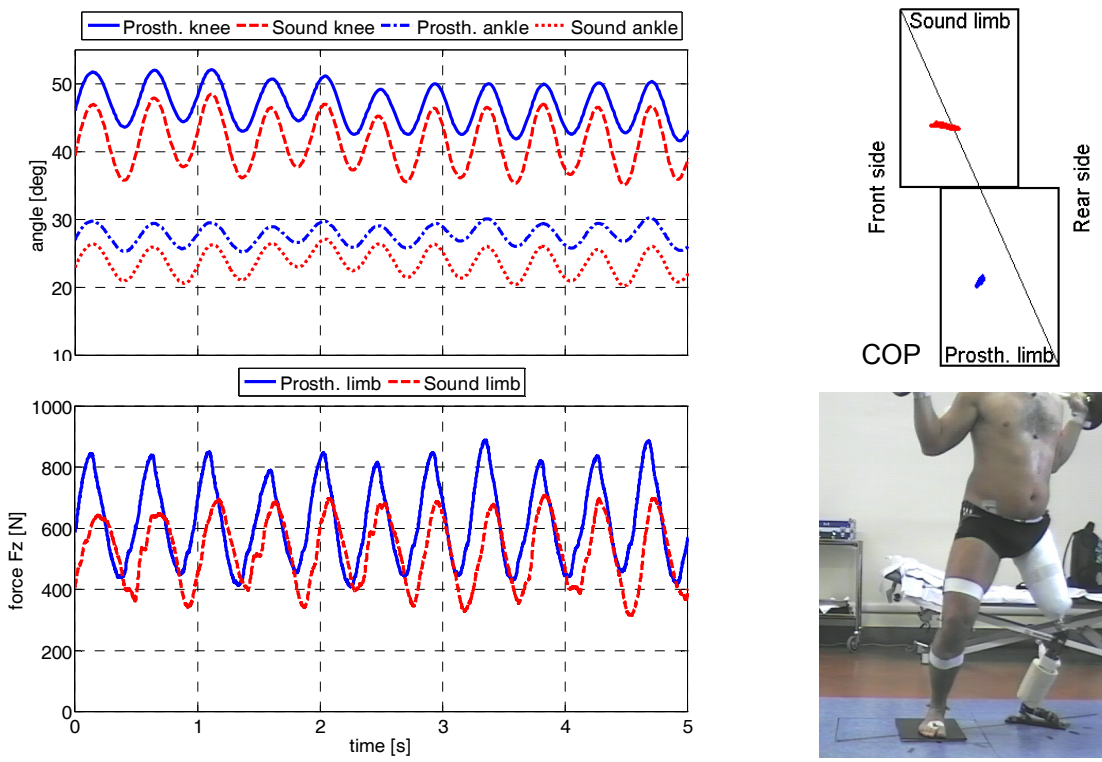

Figure 9: Amputee subject. Test conditions: high frequency, applied load and central feet position.

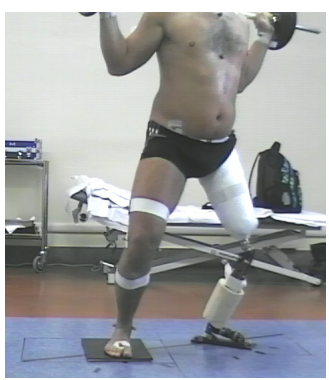


Test were performed also with the opposite feet position (Figure 2 "right position"); for sake of brevity results are omitted. However this position corresponds to a goofy one and is not favourable for the prosthesis as to load it is necessary to shift back the body weight. This force the subject to an unnatural posture, the rear limb rests only on the tip of the plate and balance is seriously jeopardised.

Last trends (Figure 9) are relative to an increased load of $180 \mathrm{~N}$. Compatibly with the fact that in this case the subject squatted with a little lower frequency than in the standard test, no meaningful differences can be pointed out.

\section{Conclusions}

Test motion analysis results showed for the able body subject, both for the kinematic and force data, a good symmetry between the legs substantially in all the tests conditions. On the contrary trends obtained with the amputee wearing the trans-femoral prosthesis are affected by the working conditions.

Stating beforehand that the amputee normally uses this prosthesis to snowboard and hence he already had it tuned and aligned to fulfil his requirements; from test results a good kinematic symmetry can observed for high frequency movements. Although the user had to maintain the load not equally distributed on the two limbs by throwing off centre the body centre of mass.

This behaviour is due to the fact that the user cannot control separately the knee and the ankle flexo-extension angles and hence the only way he has to flex them is to unbalance his body to load the prosthesis. The presence of the pneumatic system in the prosthesis has the task of helping the joint extension by returning the elastic energy stored during flexion. This explains the necessity of the user to load and unload the prosthetic limb; only for high frequency inertia actions help this process, while when moving at low frequency it is necessary to alternatively shift the centre of mass from one leg to the other. This behaviour is highlighted by the ground reaction forces trends, in which for low frequency they are in opposite phase while for high frequencies the ground reaction force on the sound limb is lower than the one measured on the controlateral.

Considering the centre of pressure graphs, generally the application point of the vertical forces on the prosthetic limb is concentrated in the anterior part of the sole. This is due to the design of the prosthesis, in fact loading it on the more tip allows to augmenting the force arm and hence the flexion moments.

\section{Acknowledgements}

The research was supported by the Fondazione Cassa di Risparmio di Torino. The authors would like to thank Gregory Leperdi and Bruno Larocca for their availability during tests. 


\section{References}

[1] Delorme S., Tavoularis S. and Lamontagne M. Kinematics of the Ankle Joint Complex in Snowboarding, J. Appl. Biomech, 21, 394-403, 2005.

[2] Klous M., Schwameder H. and Müller E. Joint loading on the lower extremities in skiing and snowboarding: methodological procedure, J Biomech 39 Suppl. 1, S187, 2006.

[3] McAlpine P.R. and. Kersting U.G. Jump Landings in Snowboarding: An Observational Study. In Proc. of 4th Int. Cong. on Science and Skiing, Austria, 2007.

[4] Krüger A. and Edelmann-Nusser J. Biomechanical analysis in freestyle snowboarding: application of a full-body inertial measurement system and a bilateral insole measurement system. Sports Technology, 1-2, 17-23, 2009.

[5] Maxwell S.M. and Hull M.L. Measurement of strength and loading variables on the knee during Alpine Skiing J. Biomech 22, 609-624, 1989.

[6] Quinn T.P. and Mote C.D. Jr. Prediction of the loading along the leg during snow skiing J Biomech 25, 609-625, 1992.

[7] Read L. and Herzog W. External Loading at the Knee Joint for Landing Movements in Alpine Skiing. Int. J. of Sports Biomech, 8, 62 - 80, 1992.

[8] Nachbauer W., Kaps P., Nigg, B.M, Brunner F., Lutz A., Obkircher G. and Mössner M. A video technique for obtaining 3-D coordinates in alpine skiing, J. Appl. Biomech., 12, 104-115, 1996.

[9] Davis 3rd R.B., Ounpuu S, Tyburski D., Gage J.R. A gait analysis data collection and reduction technique. Human Movement Science, 5, vol. 1991. 